\title{
Successful Surgical Treatment of Traumatic Macular Hole With Total Rhegmatogenous Retinal Detachment in a Child- a Case Report
}

\section{Sławomir Cisiecki}

Julianow Medical Center

Karolina Boninska ( $\nabla$ karolina.boninska@gmail.com )

Julianow Medical Center https://orcid.org/0000-0002-9856-8790

Maciej Bednarski

Julianow Medical Center

\section{Case report}

Keywords: blunt ocular trauma, macular hole, retinal detachment

Posted Date: May 13th, 2021

DOI: https://doi.org/10.21203/rs.3.rs-506882/v1

License: (c) (i) This work is licensed under a Creative Commons Attribution 4.0 International License.

Read Full License 


\section{Abstract}

Background: To investigate the surgical results and morphologic characteristics of pediatric traumatic macular hole with total rhegmatogenous retinal detachment.

Case presentation: The 4-year-old-male patient underwent scleral buckling surgery combined with vitrectomy with the inverted ILM flap technique and silicon oil tamponade for five months. Complete ophthalmic examination was performed preoperatively and 7 days, 1, 3, 6, 9 and 12 months after surgery. Successful retinal reattachment was achieved and the macular hole was successfully closed. Visual acuity improved from Hand Motion at the initial visit to 20/80 (0.6 LogMAR) postoperatively.

Conclusions: Scleral buckling surgery and vitrectomy with the inverted ILM flap technique appeared to give effective anatomical and functional results in this case.

\section{Summary Statement:}

We report the case of a 4-year-old-boy presented after direct blunt ocular trauma, resulting in a fullthickness macular hole with total retinal detachment. VA was HM. Scleral buckling surgery, with vitrectomy and the inverted ILM flap technique was performed. Anatomical and functional improvement were achieved. Final BCVA 20/80.

\section{Background:}

Retinal detachment associated with macular hole (MHRD) occurs predominantly in highly myopic eyes and staphyloma but also has been noted after blunt ocular trauma [1-4]. Although its pathogenesis remains uncertain, it is believed that MHRD can be created via two pathomechanisms. Firstly, the macular hole may allow liquified vitreous into the subretinal space and lead to retinal detachment. The retinal detachment is located in the posterior pole and may spread forward without accompanying tears. In the second suggested pathomechanism, the retinal detachment, starting from a tear or tears in the circumference, spreads backwards. Stretching the tissues over the macula leads to the development of the macular hole. It may also arise from anterior-posterior vitreoretinal tractional forces $[5,6]$.

In MHRD, vitrectomy with internal limiting membrane (ILM) peeling has become the effective surgical approach [7-10]. The potential risk of postoperative cataract formation is acknowledged [11]. When the patient is a child, choosing the correct surgical approach is especially challenging because of the risk of amblyopia.

This paper presents a rare case of successfully surgically treated pediatric full-thickness macular hole associated with total retinal detachment and dialysis.

\section{Case Presentation:}


Two days after blunt trauma to the right eye, a 4-year-old male patient presented in our ophthalmic clinic. The history of previous intraocular procedures and general diseases was negative.

A full ophthalmic examination with indirect biomicroscopy was performed. The visual acuity (VA) in the right eye was Hand Motion (20/4000; LogMAR 2.3) and was 20/25 (LogMAR 0.04) in the left eye. The intraocular pressure measured by palpation was within normal range. No abnormalities were found in the anterior segment of either eye, nor in the fundus examination of the left eye. The fundus examination of the right eye showed a full-thickness macular hole (FTMH) and upper temporal dialysis with total retinal detachment in all four quadrants. No choroidal rapture was detected.

SS-OCT (Swept source OCT), (DRI OCT Triton plus, Topcon, Japan) examination and eye ultrasonography were performed (Fig. 1, 2)

The patient was admitted to the ophthalmic department for surgical treatment.

The procedure was performed under combined intravenous-volatile anesthesia.

The eye was encircled with silicone band type 41 after placing mattress suture 6/0 Ethilon in four quadrants without tying. 23-gauge, 3-port traditional core and peripheral vitrectomy were then performed. Perifoveal posterior vitreous detachment (PVD) was observed. The ILM was stained for 1 minute with Membrane Blue dye (DORC, Rotterdam, the Netherlands). Perfluorocarbon (PFCL) (Perfluorodecalin, FDecalin, Fluoron $\mathrm{GmbH}$, Germany) was introduced and the inverted ILM flap technique was performed [12]. The ILM was grasped under PCFL during circumferential peeling and left attached to the edges of the macular hole and then inverted so that it was laying over the hole on the surface of the retina.

A small peripheral retinotomy was performed over the PCFL bubble to facilitate the subretinal fluid/air exchange. PVD was not forced to the periphery because of potential risk of iatrogenic breaks and was therefore finished at the temporal arcades. Extensive peripheral vitrectomy was avoided so as not to touch the lens and cause cataract.

After fluid/air exchange the eye was completely filled with PCFL, which was then exchanged for $5000 \mathrm{cs}$ silicone oil. The $360^{\circ}$ buckle was then sutured in order to close retinal dialysis, whilst carefully monitoring intraocular pressure. Sclerotomies were closed with Nylon 10/0 and conjunctiva with 7/0 Vicryl.

Successful macular hole closure was achieved and noted at the first control one week after surgery. The retina was reattached (Fig. 3). The silicon oil was removed after five months. Postoperatively, fovea architecture gradually improved and BCVA also showed improvement (Fig. 4a-e). Final VA improved to 20/80 (0.6 LogMAR). In the follow-up period (currently 12 months) no cataract formation has been observed.

\section{Discussion And Conclusion:}


The most difficult aspect of the described case was determining how to attain retinal reattachment and macular hole closure at the same time. The $87,5 \%$ success rate of scleral buckling in total retinal detachment caused by retinal dialysis is very high [13]. Moreover, traumatic full-thickness macular holes can close spontaneously with time [14-16]. However, in our experience this can take 6 months or more, which is unacceptable in a child because of the risk of amblyopia. Additionally, the early observation of perifoveal PVD and large macular hole diameter in this case reduced the chances of spontaneous closure [14-16].

In this particular case, we had to choose between retinal reattachment only, with just a segmental or 360 buckle in the hope that the FTMH would close spontaneously at some time. Or we could attempt to close the macular hole and reattach the retina in a single procedure by combining vitrectomy and scleral buckling. Choosing the second option also meant that we did not need to perform complete vitrectomy. The anterior vitreous was left and the risk of cataract formation was therefore decreased [11]. The inverted ILM flap technique is a novel surgical technique that was reported to enhance closure of complicated cases of macular hole [12]. As it is a technique routinely used in our department, and covering macular holes with the inverted ILM flap has given us a $100 \%$ success rate, we felt confident that it was suitable method in this case. Silicone oil was used due to the patient's inability to maintain prone position for any length of time.

In conclusion, our experience in this single case suggests that in pediatric MHRD, scleral buckling with vitrectomy enables good anatomical and functional results. In 12 months of follow-up, no redetachment has been seen, the $\mathrm{MH}$ remains closed and there are no signs of cataract formation.

\section{Abbrevations:}

RD- retinal detachment

VA- visual acuity

HM- hand movement

ILM- Internal Limiting Membrane

BCVA- Best-corrected visual acuity

MHRD- Retinal detachment associated with macular hole FTMH- full-thickness macular hole SS-OCT-Swept source OCT

PVD- posterior vitreous detachment $\mathrm{MH}$-macular hole

PFCL-Perfluorocarbon liquid

Fig.- figure 


\section{Declarations:}

\section{Ethics approval and consent to participate:}

Written informed consent was obtained from the patient's legal guardian(s) for publication of this case report and any accompanying images. A copy of the written consent is available for review by the Editorin-Chief of this journal.

\section{Consent to publish:}

Written informed consent for publication of their clinical details and clinical images was obtained from the relative of the patient. A copy of the consent form is available for review by the Editor of this journal.

\section{Availability of data and materials:}

All data generated and analysed during this study are included in this published article

\section{Competing of interest:}

There is no conflict of interest

\section{Funding:}

$\mathrm{N} / \mathrm{A}$

\section{Authors' Contributions:}

SC: conceived and designed the analysis, performed the analysis; KB: wrote the paper; MB: collected the data.

All Authors read and approved the manuscript.

\section{Acknowledgements:}

Hindawi Editig Editage - professional editing service

\section{References:}

1. Siam A. Macular hole with central retinal detachment in high myopia with posterior staphyloma. $\mathrm{Br} \mathrm{J}$ Ophthalmol 1969;53: 62-63. DOI: 1136/bjo.53.1.62.

2. Stirpe M, Michels RG. Retinal detachment in highly myopic eyes due to macular holes and epiretinal traction. Retina 1990; 10:113-114. DOI: 1097/00006982-199004000-00004.

3. Ohsugi $\mathrm{H}$, Ikuno $\mathrm{Y}$, Matsuba S, et al. Morphologic characteristics of macular hole and macular hole retinal detachment associated with extreme myopia. 2019 Jul;39(7):1312-1318.

DOI: 10.1097/IAE.0000000000002155 
4. Lim LS, Tsai A, Wong D et al. (2014): Prognostic factor analysis of vitrectomy for retinal detachment associated with myopic macular holes. Ophthalmology 121: 305-310.

DOI: 1016/j.ophtha.2013.08.033

5. Wu AL, Ling KP, Chuang LH, et al. Treatment of macular hole retinal detachment with macular plug in highly myopic eyes: three-year results. Acta Ophthalmol.2020 Apr 3. doi: 10.1111/aos.14418. [Epub ahead of print] DOI: $1111 /$ aos. 14418

6. Morita $\mathrm{H}$, Ideta $\mathrm{H}$, Ito $\mathrm{K}$, et al. Causative factors of retinal detachment in macular holes. Retina.1991;11:281-284. DOI: 1097/00006982-199111030-00002

7. Oie Y, Emi K, Takaoka G, Ikeda T. Effect of indocyanine green staining in peeling of internal limiting membrane for retinal detachment resulting from macular hole in myopic eyes. Ophthalmology. 2007;114:303-306. DOI: 1016/j.ophtha.2006.07.052

8. Ripandelli G, Coppe AM, Fedeli R, Parisi V, D'Amico DJ, Stirpe M. Evaluation of primary surgical procedures for retinal detachment with macular hole in highly myopic eyes: a comparison [corrected] of vitrectomy versus posterior episcleral buckling surgery. Ophthalmology. 2001;108:2258-2264. DOI: 1016/s0161-6420(01)00861-2

9. Lim LS, Tsai A, Wong D, et al. Prognostic factor analysis of vitrectomy for retinal detachment associated with myopic macular holes. Ophthalmology. 2014;121:305-310.

DOI: 1016/j.ophtha.2013.08.033

10. Seike C, Kusaka S, Sakagami K, Ohashi Y. Reopening of macular holes in highly myopic eyes with retinal detachments. Retina. 1997;17:2-6. DOI: 1097/00006982-199701000-00001.

11. Petermeier K, Szurman P, Bartz-Schmidt UK. Gekele F. Pathophysiologie der Katarakt-Entwicklung nach Vitrektomie. Klin Monbl Augenheilkd 2010; 227(3): 175-180. DOI: 1055/s-0029-1245271.

12. Michalewska Z, Michalewski J, Adelman RA, Nawrocki J. Inverted internal limiting membrane flap technique for large MH. Ophthalmology. 2010;117(10):2018-2025. DOI: 1016/j.ophtha.2010.02.011.

13. Chang JS, Marra K, Flynn HW Jr, Berrocal AM, Arroyo JG. Scleral Buckling in the Treatment of Retinal Detachment Due to Retinal Dialysis. Ophthalmic Surg Lasers Imaging Retina. 2016;47(4):336-40. DOI: 3928/23258160-20160324-06

14. Yamashita T, Uemara A, Uchino E, et al. Spontaneous closure of traumatic macular hole. American Journal of Ophthalmology. 2002;133(2):230-235. DOI: 1016/s0002-9394(01)01303-4.

15. Mitamura $Y$, Saito $W$, Ishida $M$, et al. Spontaneous closure of traumatic macular hole. Retina. 2001;21(4):385-389. DOI: 1097/00006982-200108000-00020

16. de Filippi Sartori J, Stefanini F, de Moraes NSB. Spontaneous closure of pediatric traumatic macular hole: case report and spectral-domain OCT follow-up. Arquivos Brasileiros de Oftalmologia. 2012;75(4):286-288. DOI: 1590/s0004-27492012000400015

\section{Figures}




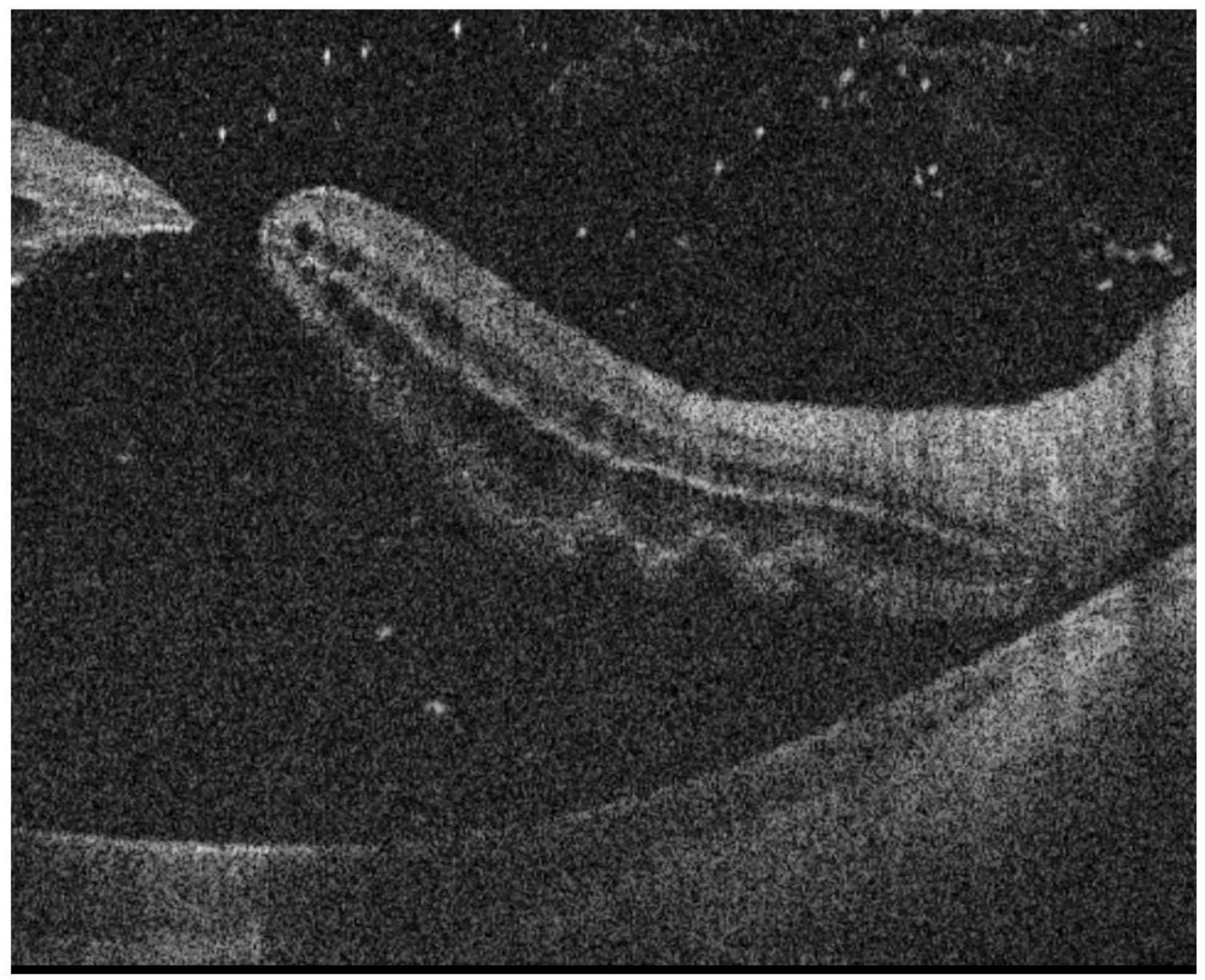

Figure 1

a-b. Preoperative Swept-Source OCT images demonstrate macular hole with PVD and total retinal detachment. 


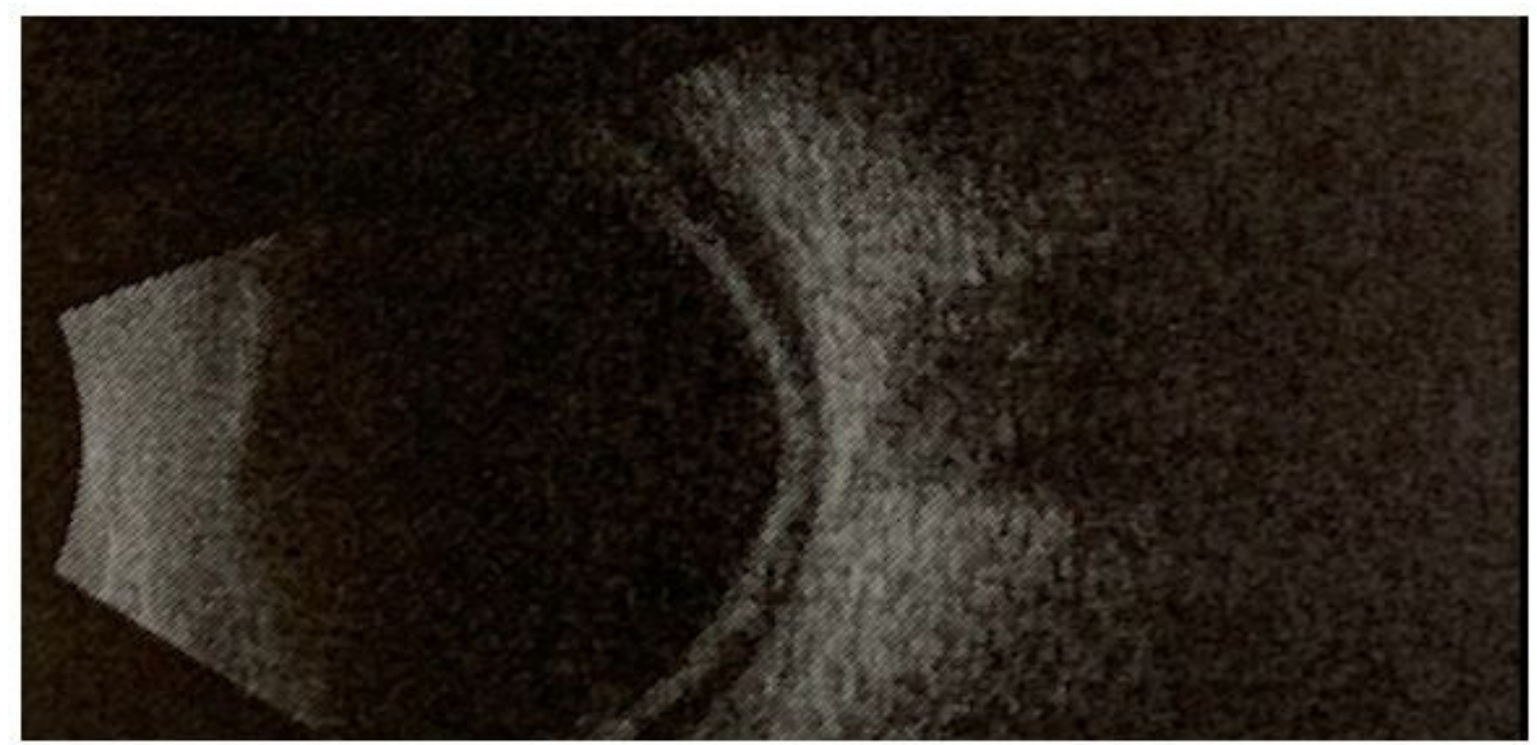

Figure 2

B mode ultrasound imaging - total retinal detachment. 


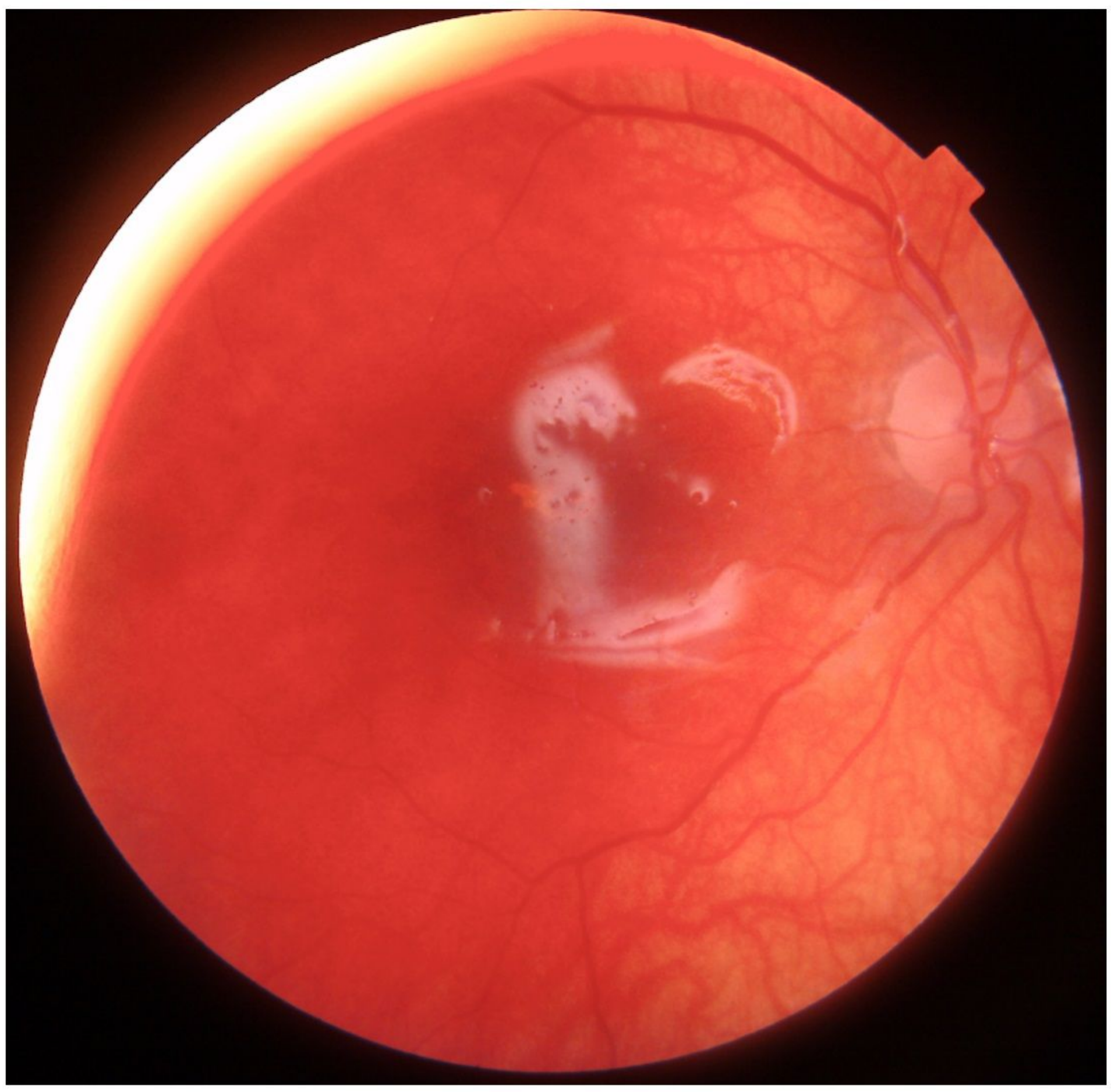

\section{Figure 3}

Color fundus photography demonstrates retinal reattachment with silicone oil tamponade, 2 months after surgery. 


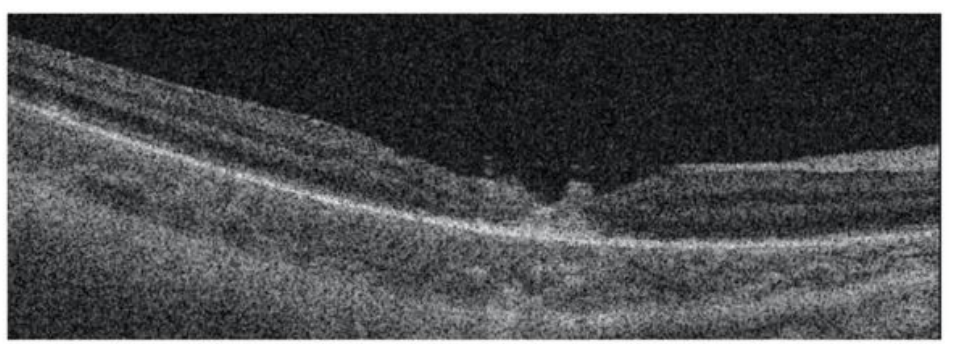

$4 a$

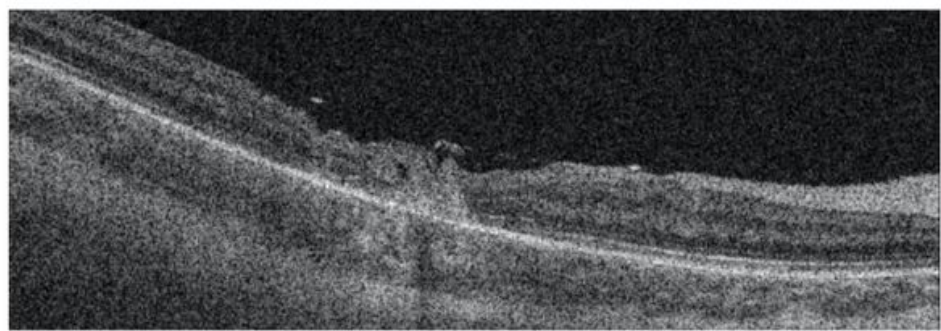

$4 c$

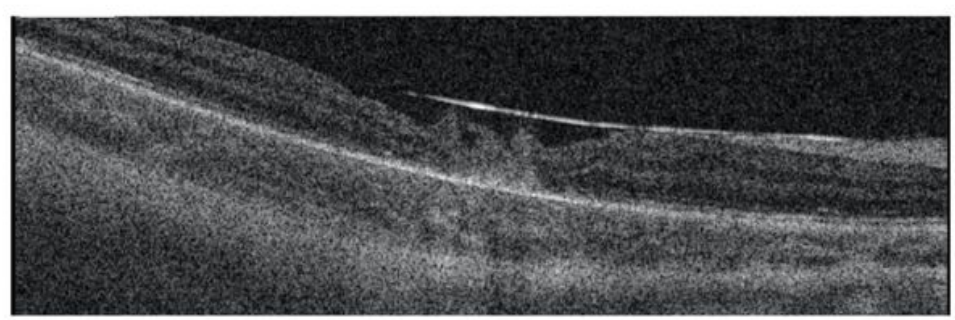

$4 b$

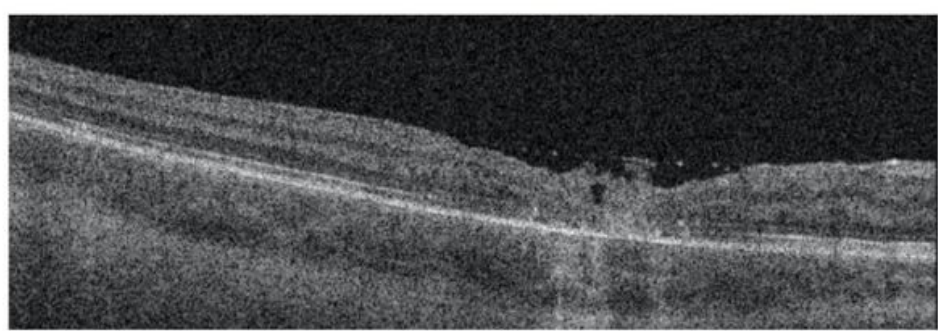

$4 d$

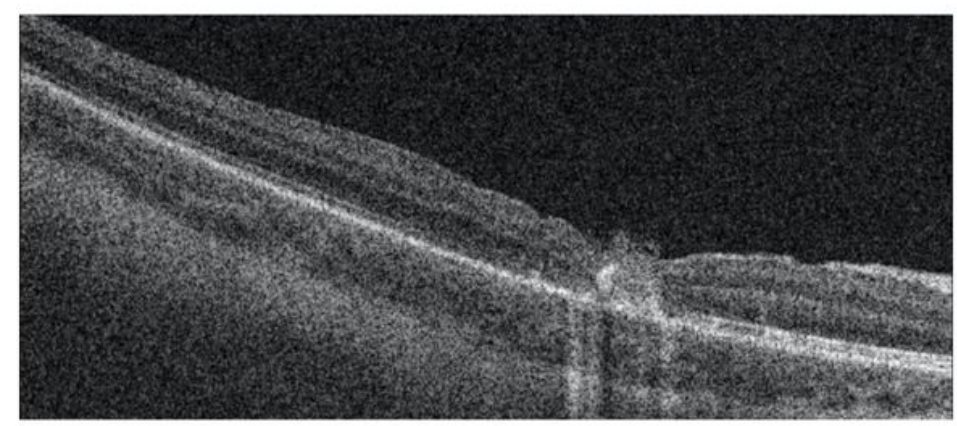

$4 e$

\section{Figure 4}

Postoperative Swept-Source OCT images. (A) 2 weeks after surgery, BCVA=20/200; (B) 1 month postoperatively $B C V A=20 / 200$; (C) 3 months postoperatively, $B C V A=20 / 100$; (D) 6 months postoperatively, BCVA-20/80; (E) 1 year postoperatively $B C V A=20 / 80$. 